\title{
A Lightweight RFID Protocol Using Substring
}

\author{
Hung-Yu Chien and Chen-Wei Huang \\ Dept. of Information Management, National Chi Nan University, Taiwan, R.O.C. \\ \{hychien, s95213508\} @ncnu.edu.tw
}

\begin{abstract}
As low-cost RFIDs with limited resources will dominate most of the RFID market, it is imperative to design lightweight RFID authentication protocols for these low-cost RFIDs. However, most of existing RFID authentication protocols either suffer from some security weaknesses or require costly operations that are not available on low-cost tags. In this paper, we analyze the security vulnerabilities of a lightweight authentication protocol recently proposed by Li et al. [4], and then propose a new lightweight protocol to improve the security.
\end{abstract}

Keywords: RFID, authentication, low-cost cryptography, tracing, reader, DOS attack.

\section{Introduction}

A Radio Frequency Identification (RFID) system mainly consists of three components: radio frequency tags, readers, and a backend server/database (or a set of distributed databases) which maintains information on the tagged objects. Generally, the tag consists of a microchip with some data storage and an antenna. A reader queries tags to obtain tag contents though wireless communications.

Recently, the wide deployment of RFID systems in a variety of applications has raised many concerns about the privacy and the security. An RFID tag can be attached to a product, an animal, or a person for the purpose of identification using radio waves. For any possible reasons, an adversary may perform various attacks such as eavesdropping, traffic analysis, spoofing, disabling the service, or disclosing sensitive information of tags, and hence infringes people's privacy and security.

Even though RFID tags with full-fledged capacity are available, to attain great market penetration, RFID tags should be low-cost, which limit the computation power, the storage space, the communication capacity and the gates count. As studied by the previous work like [2], a low-cost RFID tag has approximately 4,000 logic gates. Although there have been many works devoted to design security mechanisms for low-cost RFIDs, most of these works require the tags to be equipped with costly operations such as one-way hashing functions $[1,3,5]$, which are still un-available on low-cost tags. Contrary to these works, the schemes $[4,6,8,9]$ do not require the support of hashing functions on tags. However, the schemes [6, 8, 9] have been reported to show some security weaknesses [6, 7]. Recently, Li et al. [4], based on only bitwise XOR $(\oplus)$, the Partial ID concept and pseudo random numbers, proposed a lightweight RFID authentication protocol for low-cost RFIDs. Different from most 
of existing solutions like $[1,3,5]$ which used conventional cryptographic primitives (encryptions, hashing, etc), this protocol only used simple operations like XOR and substring. Unfortunately, we find that Li et al.'s scheme has several security weaknesses. In this paper, we shall analyze the security weaknesses of Li et al.'s RFID authentication protocol. To heal the weaknesses while preserving the lightweight feature, we propose a new RFID authentication protocol.

The rest of this paper is organized as follows. Section 2 reviews Li et al. lightweight RFID authentication protocol. Section 3 analyzes the vulnerabilities of Li et al.'s scheme. Section 4 proposes a new RFID authentication protocol that heals the security weaknesses while preserving the lightweight feature for low-cost RFID tags. Section 5 analyzes the security of our proposed protocol. Finally, conclusion remarks and future work are drawn in Section 6.

\section{Review of Li et al.'s Scheme}

The tags in Li et al.'s RFID authentication protocol [4] use only bitwise XOR $(\oplus)$, the partial ID concept and pseudo random numbers. Costly operations such as multiplications and hash functions are eliminated in the design. In Li et al.'s scheme, each tag and the backend server share an $l$-bit secret information, SID (the secure ID). During the authentication the tag generates two random numbers $n_{l}$ and $n_{2}$ such that $2 l \geqq n_{1}+n_{2} \geqq l / 2$. The two random numbers are used in the substring function $f$ to extract the partial $I D$ s, $P I D_{1 L}$ and $P I D_{2 R}$, where $P I D_{I L}$ denotes the left substring of $S I D$ and $P I D_{2 R}$ denotes the right substring of SID. That is, let $f(S I D, i, j)$ denotes the substring of $S I D$ starting from position $i$ to position $j$, then $P I D_{I L}=f\left(S I D, 1, n_{l}\right)$ and $P I D_{2 R}=f\left(S I D, n_{2}, l\right)$. Li et al.'s scheme is depicted in figure 1 .

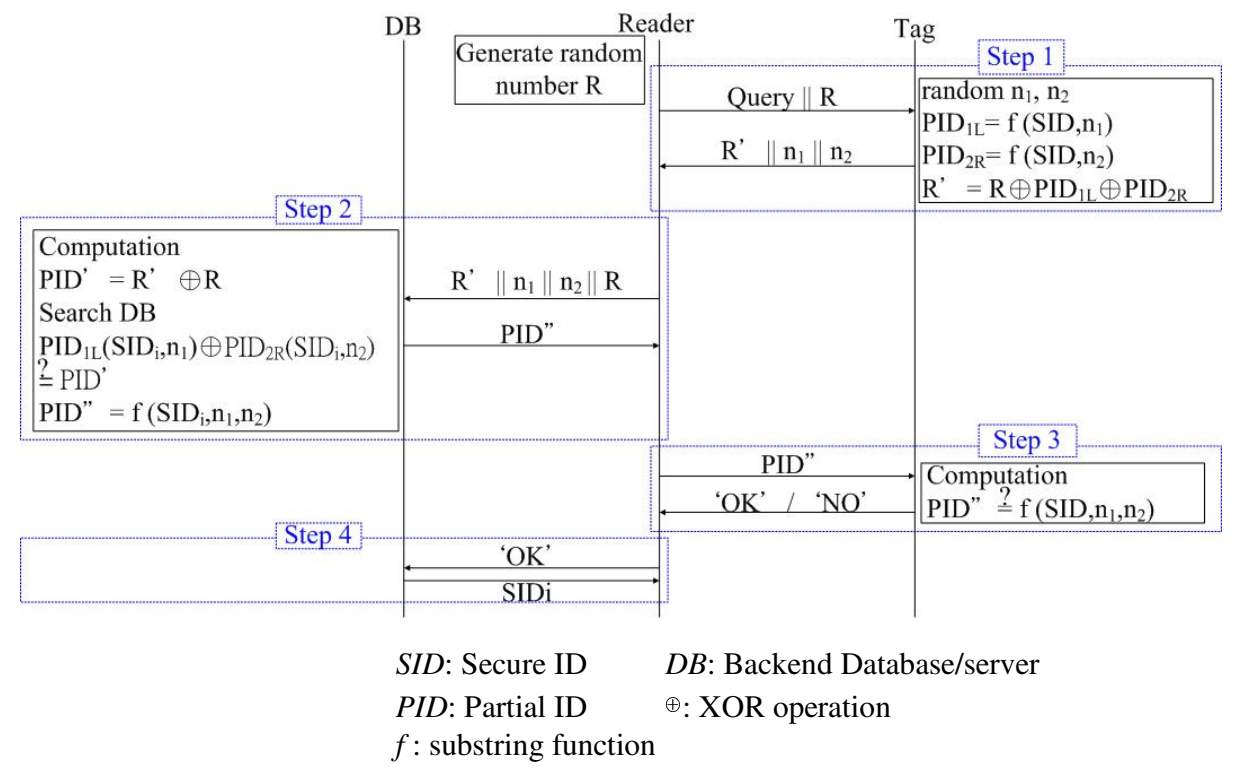

Fig. 1. Li et al.'s scheme 
The scheme consists of four stages: the PID generating stage, the SID searching and tag authentication stage, the reader authentication stage and the result returning stage.

- PID generating stage: The reader generates a random number $R$, and then sends it to the tag. Upon receiving the probe from the reader, the tag uses two random numbers $n_{1}, n_{2}$ and the substring function $f$ to compute $P I D_{1 L}=f\left(S I D, 1, n_{1}\right), P I D_{2 R}$ $=f\left(S I D, n_{2}, l\right)$ and $R^{\prime}=R \oplus P I D_{l L} \oplus P I D_{2 R}$. The tag then responds the data $R^{\prime}, n_{l}$ and $n_{2}$ to the reader.

- SID searching and tag authentication stage: The reader sends $R^{\prime}, R, n_{l}$ and $n_{2}$ to the server. The server computes $P I D^{\prime}=R^{\prime} \oplus R$, and iteratively picks up one candidate $S I D$ ' from the database to check whether $P I D^{\prime}{ }_{I L} \oplus P I D{ }_{2 R}=P I D$, where $P I D{ }^{\prime}{ }_{1 L}=f\left(S I D ', 1, n_{l}\right)$ and $P I D{ }_{2 R}=f\left(S I D ', n_{2}, l\right)$. If a match is found, then the selected $S I D$ ' is the tag's identification; otherwise, it continues the process until a match is found or responds with "failure" if no match could be found in the whole database. If a match is found, it computes $P I D$ ” $=f\left(S I D\right.$ ', $\left.n_{1}, n_{2}\right)$ and then sends it to the reader.

- Reader authentication stage: The reader sends PID" to the tag, which then checks whether $f\left(S I D, n_{1}, n_{2}\right)$ equals $P I D$ ” to authenticate the reader. After the reader is authenticated successfully, the tag sends 'OK' to the reader; otherwise, it responds with "no find" information.

- Result returning stage: If the reader receives 'OK', and then sends it to the server, which will transmit the SID to the reader. Otherwise the reader stops the protocol.

\section{Vulnerabilities of Li et al. Scheme}

In this section, we remark that Li et al.'s scheme is vulnerable to replay attack and is prone to reveal the secret information SID.

\subsection{The Replay Attack}

An adversary can easily eavesdrop on the communications from a legal tag, modify the data, and then replay the messages to masquerade as the legal tag as follows. The attack consists of two stages- the data deriving stage and the spoofing stage.

- The data deriving stage: The adversary records the communication $\left(R, R^{\prime}, n_{l}\right.$, $n_{2}$ ) from a tag (say $T_{a}$ ), and then derives $P I D_{I L} \oplus P I D_{2 \mathrm{R}}$ from $R^{\prime} \oplus R$.

- The spoofing stage: In this stage, the adversary uses the derived data $P I D_{1 L} \oplus P I D_{2 \mathrm{R}}$ to masquerade as the tag $T_{a}$ as follows.

1. Upon receiving the probe Queryll $\bar{R}$ from the reader, the adversary computes $R^{\prime}=P I D_{1 L} \oplus P I D_{2 R} \oplus \bar{R}$, and responds with $R^{\prime}\left\|n_{1}\right\| n_{2}$ to the reader.

2. It is easy to see that the forged data $R^{\prime}\left\|n_{1}\right\| n_{2}$ will be accepted by the server, and the reader will forward the data $P I D "$ from the server to the adversary. 
3. The adversary just records the data $P I D$ " and always responds with "OK" to the reader. We can see that the reader finally accepts this spoofing tag as the genuine tag $T_{a}$.

\subsection{Disclosing the Secret Value SID}

Since an adversary can eavesdrop on the communications and record the data $R^{\prime}, R$, $n_{l}, n_{2}$ and $P I D$ ”, and he can compute $R^{\prime} \oplus R$ to obtain $P I D_{I L} \oplus P I D_{2 R}$. With $n_{l}, n_{2}, R^{\prime} \oplus R$ and $P I D$ ", an adversary can derive partial information of $S I D$, and can repeatedly run the process many times to fully disclose all the bits of SID or derive partial information of SID (if most bits of the identification are known, then it is highly possible to guess the rest bits because the identification of a tag-for example, the EPC code- has a pre-defined format). In the following, we describe the single run of our attack process, and examine some cases to point out the vulnerabilities of Li et al.'s scheme.

As the lengths of $P I D_{1 L}, P I D_{2 R}$ and $P I D$ ” are unequal to $l$ bits, we assume that $0 \mathrm{~s}$ are padded to them such that each length of them equals $l$-bit in the following scenario (we can also assume $1 \mathrm{~s}$ are padded to these strings, and the same attack still works). Based on the values $R^{\prime} \oplus R=P I D_{1 L} \oplus P I D_{2 R}$ and $P I D "=f\left(S I D, n_{1}, n_{2}\right)$, an adversary can derive parts of $S I D$. The length of the disclosed part of $S I D$ depends on the values of $n_{l}$ and $n_{2}$. With $2 l \geqq n_{l}+n_{2} \geqq l / 2$ property, the values of $n_{l}$ and $n_{2}$ generally have four situations. Firstly, if $n_{l}=l-n_{2}$, an adversary can derive $f\left(S I D, n_{l}, n_{2}\right)$. Secondly, if $n_{l}>$ $l-n_{2}$, an adversary can derive $f\left(S I D, n_{l}, n_{2}\right)$ and $f\left(S I D, l-n_{2}, n_{l}\right)$. Thirdly, if $n_{l}<l-n_{2}$, an adversary can derive $f\left(S I D, n_{l}, n_{2}\right)$ and $f\left(S I D, n_{l}+n_{2}, l\right)$. Finally, if $n_{l}=l, l-n_{2}=0$, an adversary can obtain all of SID. Some example cases are discussed as follows.

Example 1. Deriving parts of SID. Assume $n_{l}=l / 4$ and $n_{2}=3 / 4 l$, an adversary can directly derive the $l / 2$-bits $f\left(S I D, n_{1}, n_{2}\right)$ from $P I D$ ”.

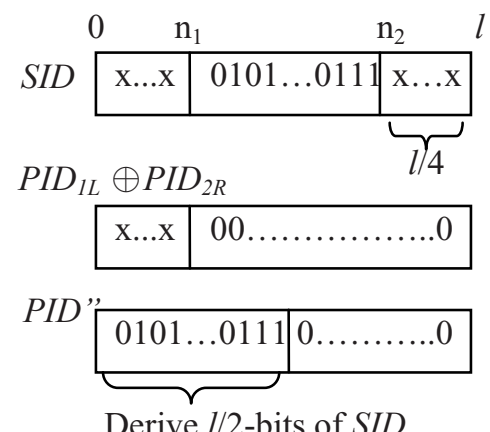

Example 2. Deriving parts of $\boldsymbol{P I D}_{1 L}$. Assume $n_{l}=l / 2$ and $n_{2}=3 / 4 l$, an adversary can derive $l / 4$-bit $f\left(S I D, l-n_{2}, n_{l}\right)$ from $P I D_{l L} \oplus P I D_{2 R}$, and derive $l / 4$-bit $f\left(S I D, n_{l}, n_{2}\right)$ from $P I D ”$ as follows. 

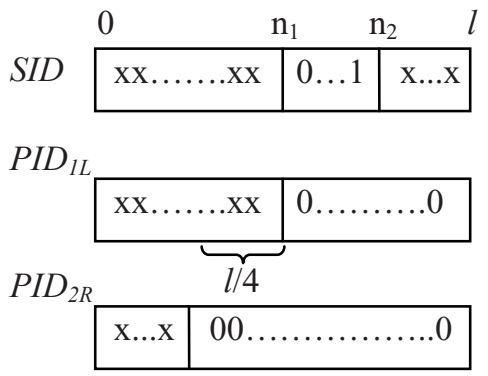

$P I D_{1 L} \oplus P I D_{2 R}$

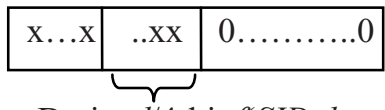

Derive $l / 4$-bit $f\left(S I D, l-n_{2}, n_{l}\right)$

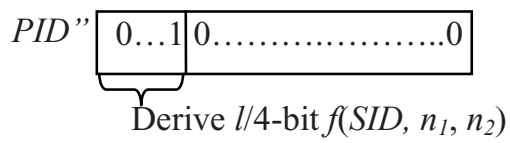

Example 3. Deriving parts of $\boldsymbol{P I D}_{2 \boldsymbol{R}}$. Assume $n_{l}=1 / 3 \quad l$ and $n_{2}=l / 2$, an adversary derives $l / 6$-bit $f\left(S I D, n_{l}+n_{2}, l\right)$ from $P I D_{l L} \oplus P I D_{2 R}$, and derive $l / 6$-bit $f\left(S I D, n_{l}, n_{2}\right)$ from $P I D ”$ as follows.
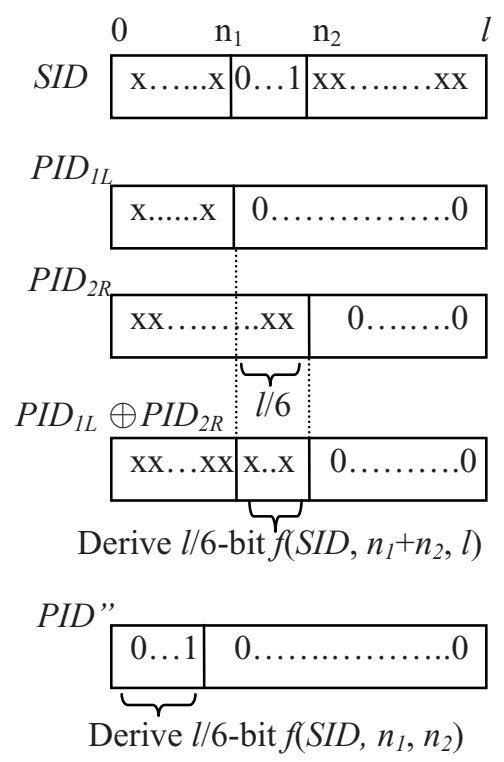

Example 4. Deriving all the bits of SID. Assume $n_{l}=l$ and $n_{2}=l$, an adversary can obtain all the bits of SID as follows. 


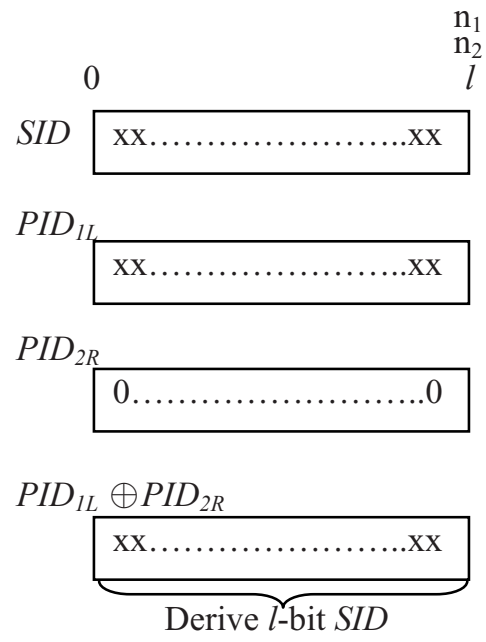

In a single run of the above attack, an adversary can derive partial information of $S I D$, and he can launch the above attack several times to aggregate the partial information of SID or even derive all the bits of SID. Although it is possible that part of the SID can not be directly derived in the above process, one might guess the rest bits, because the identifications of tags are usually with fixed format.

\section{A New Lightweight RFID Authentication Protocol}

In this section, we propose a new protocol to improve the security while preserving the lightweight property. Our proposed protocol is depicted in figure 2 and described as follows.

We assume that each tag and the database share an $l$-bit secret key $x, x=x_{0} x_{1} \ldots \ldots x_{l-}$ ${ }_{l} x_{l}$. The reader generates a random number $R_{l}$, and the tag generates a random number $R_{2}$. Some notations are introduced as follows.

$g(z): g()$ is a random number generator, and $z$ is an input number.

$\tilde{g}:$ the random output of $g(z)$.

rotate $(p, w)$ : rotate denotes the bitwise left rotation operator, and the operand $p$ is rotated $w$ positions.

Left $(s)$ : the left half of $s$.

$\operatorname{Right}(s)$ : the right half of $s$.

Step1: The reader generates a random number $R_{l}$, and then sends it to the tag. Upon receiving the probe from the reader, the tag generates another random number $R_{2}$, computes $\tilde{g}=g\left(R_{1} \oplus R_{2} \oplus x\right)$ and rotates its $S I D$ to obtain $S^{\prime} D^{\prime}=$ rotate $(S I D, \tilde{g})$. It calculates $R^{\prime}=\operatorname{Left}\left(S I D^{\prime} \oplus \tilde{g}\right)$, and responds the data $R^{\prime}$ and $R_{2}$ to the reader. 


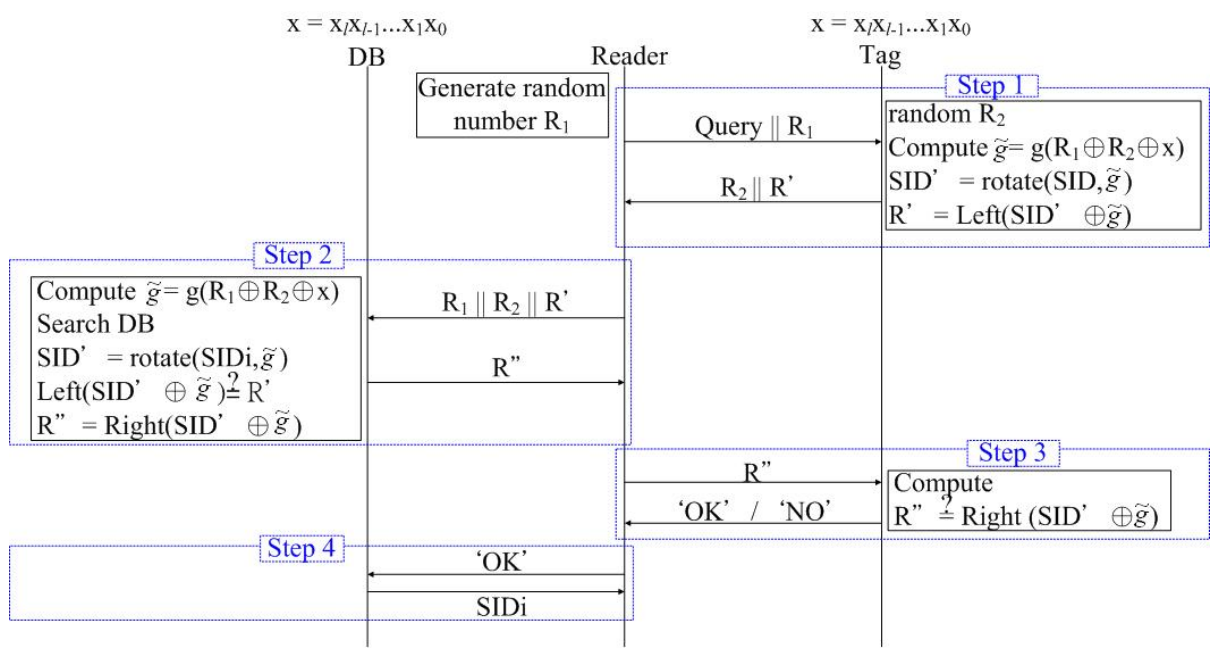

Fig. 2. Our proposed protoco1

Step2: The reader forwards $R_{1}, R_{2}$, and $R^{\prime}$ to the server. The server iteratively picks up one candidate $S I D$ from the database, computes $\tilde{g}=g\left(R_{1} \oplus R_{2} \oplus x\right)$ and $S I D^{\prime}=$ rotate $(S I D, \tilde{g})$ and checks whether $\operatorname{Left}\left(S I D^{\prime} \oplus \tilde{g}\right)=R^{\prime}$. If a match is found, then the selected SID is taken as the tag identification; otherwise, it continues the process until a match is found or responds with "failure"" if it cannot find a match in the whole database. If a match is found, it computes $R$ " $=\operatorname{Right}\left(\operatorname{SID}^{\prime}\right.$ $\oplus \tilde{g}$ ) and then sends it to the reader.

Step3: The reader sends $R$ ” to the tag, which then checks whether $\operatorname{Right}\left(\operatorname{SID}^{\prime} \oplus \tilde{g}\right)$ equals $R$ " to authenticate the reader. After the reader is authenticated successfully, the tag sends 'OK' to the reader; otherwise, it responds with "no find" information.

Step4: If the reader receives 'OK', and then sends it to the server, which then transmits the SID to the reader. Otherwise the reader stops the protocol.

During singulation, if multiple tags respond simultaneously to a query, they will interfere with each other. Therefore, we suggest that an anti-collision algorithm like the binary tree-walking [3] could be used in our proposed protocol to solve the problem of collisions.

\section{Analysis}

\subsection{Security Analysis}

We now analyze the security of the proposed scheme as follows.

- No traceability. During each authentication instance, an adversary can only observe the values $\left(R_{1}, R_{2}, R^{\prime}, R^{\prime \prime}\right)$, where $R_{1}, R_{2}$ are random numbers and 
$R^{\prime} / R^{\prime \prime}$ are respectively the left/right half bits of the random string $S I D^{\prime} \oplus \tilde{g}$. No identity-related information can be derived from these values, and these values are distinct and look random to an adversary. So, an adversary cannot trace the tags.

- Mutual authentication. The server authenticates the tag by verifying the substring $\operatorname{Left}\left(S I D^{\prime} \oplus \tilde{g}\right)$, and the tag authenticates the server by verifying the substring $\operatorname{Right}(S I D \prime \oplus \tilde{g})$. Since only the genuine tag and the server who have the secret key $x$ can generate and verify the values, the scheme provides mutual authentication.

- Replay attack prevention. An adversary could eavesdrop on the communications between the reader and the tag. However, the substring $\operatorname{Left}(\operatorname{SID}, \oplus \tilde{g})$ and the substring $\operatorname{Right}(S I D, \oplus \tilde{g})$ should depend on the random challenges $R_{1}, R_{2}$, and replay messages cannot satisfy the verification either by the reader or by the tag.

- DOS attack prevention. In some previous schemes, the technique of varying pseudonyms is used to resist tracing, and these schemes need to synchronize the pseudonyms between the server and the tags; otherwise, they are unable to authenticate each other. In our scheme, there is no requirement of state synchronization. Therefore, it can resist the DOS attack.

In Table 1, we show a comparison of the security with previous mentioned schemes $[1,2,3,5]$.

Table 1. Comparison between schemes

\begin{tabular}{|c|c|c|c|c|c|c|}
\hline Protocol & RHLK[3] & HBIV[1] & SRAC[5] & LCAP[2] & Li et al.[4] & Our scheme \\
\hline No traceability & $\mathrm{x}$ & $\mathrm{x}$ & $\mathrm{x}$ & $\mathrm{O}$ & $\mathrm{o}$ & $\mathrm{O}$ \\
\hline Mutual Authentication & $\mathrm{o}$ & $\mathrm{o}$ & $\mathrm{o}$ & $\mathrm{o}$ & $\mathrm{o}$ & $\mathrm{O}$ \\
\hline Replay attack prevention & $\mathrm{x}$ & $\mathrm{x}$ & $\mathrm{x}$ & $\mathrm{O}$ & $\mathrm{x}$ & $\mathrm{O}$ \\
\hline DOS attack prevention & $\mathrm{x}$ & $\mathrm{o}$ & $\mathrm{x}$ & $\mathrm{x}$ & $\mathrm{o}$ & $\mathrm{o}$ \\
\hline
\end{tabular}

\subsection{Performance Analysis}

It is important to minimize the storage cost, the computational cost and the communication cost of low-cost tags. In Table 2, we examine the performance of our scheme in terms of storage space, computational cost and communication cost, and compare it with the previous schemes $[1,2,3,5]$.

Storage space: In our scheme, the tag has to store its tag $I D$ of length $l$ and an $l$ bit secret key. For identifying the tag, the database has also to store related information. Therefore, implementation of our scheme, the tag and the database both only require $2 l$ bits of memory, which is suitable to low-cost tags. 
- Computational cost: In Henrici-Müller's scheme [1], Lee et al.'s scheme [2], Weis et al.'s scheme [3] and Lee-Verbauwhede's scheme [5], the tag has to be equipped with hash functions, which are still un-available on low-cost tags. On the contrary, in our scheme, the tag only needs random number generation, $\mathrm{XOR}$, shifting, and substring function. The computations are very efficient and lightweight.

- Communication cost: In our scheme, messages of tag-to-reader communication are $R_{2}$ and $R^{\prime}$ with a total of $1 \frac{1}{2} l$ bits and a message of reader-to-tag communication is $R$ ” with $\frac{1}{2} l$ bits. Compared to Henrici-Müller's scheme [1], Weis et al.'s scheme [3] and Lee-Verbauwhede's scheme [5], the communication performance of our scheme is more efficient.

Table 2. Performance analysis

\begin{tabular}{|c|c|c|c|c|c|c|c|}
\hline \multicolumn{2}{|c|}{ Protocol } & RHLK[3] & HBIV[1] & SRAC[5] & LCAP[2] & Li et al.[4] & Our scheme \\
\hline \multirow{3}{*}{ Storage. } & Tag & $l$ & $3 l$ & $l$ & $l$ & $l$ & $2 l$ \\
\cline { 2 - 8 } & Reader & - & - & - & - & - & - \\
\cline { 2 - 8 } & Database & $n l$ & $10 l$ & $4 l$ & $6 l$ & $l$ & $2 l$ \\
\hline \multirow{3}{*}{ Comp. } & Tag & $1 h$ & $3 h$ & $2 h$ & $2 h$ & 2 (XOR) & 3 (XOR) \\
\cline { 2 - 8 } & Reader & $1 h$ & - & $2 h$ & - & - & - \\
\cline { 2 - 8 } & Database & - & $3 h$ & - & $2 h$ & 2 (XOR) & 4 (XOR) \\
\hline \multirow{2}{*}{ Comm. } & Tag-to-Reader & $2 l$ & $3 l$ & $l$ & $1 \frac{1}{2} l$ & $l+\alpha$ & $1 \frac{1}{2} l$ \\
\cline { 2 - 8 } & Reader-to-Tag & $l$ & $2 l$ & $2 l$ & $\frac{1}{2} l$ & $\beta$ & $\frac{1}{2} l$ \\
\hline
\end{tabular}

Notations of Table: $l$ - size of required memory, $h$ - the cost of a hash function operation, $\alpha-$ between $l / 2$ and $2 l, \beta-$ less than $l$.

\section{Conclusion and Future Work}

This paper has shown the replay attack and the secret disclosure problem of Li et al.'s scheme. In the attack, an adversary can easily derive partial information of the secret $S I D$ or even all the bits of the SID. We also have proposed a new lightweight RFID authentication protocol, which improves the security, the communication performance and the computational performance. And taking into account that low-cost tags are highly resource-constrained, the tags only need to store tag's $I D$ of length $l$ and an $l$ bit secret key. So it can easily be implemented on those low-cost RFIDs like EPC generation 2 RFID.

In Our proposed protocol, it doesn't offer forward secrecy since the key updating is not fulfilled after the mutual authentication. But, we find that the previous re-keying protocols like [1, 2, 5, 8, 9] all suffer from DOS attacks. Furthermore, designing a protocol that simultaneously ensures forward secrecy and DOS attack resistance is our future work.

Acknowledgments. This research is partially supported by National Science Council with project number NSC95-2221-E-260-050-MY2. 


\section{References}

1. Henrici, D., Müller, P.: Hash-based Enhancement of Location Privacy for Radio-Frequency Identification Devices using Varying Identifiers. In: PerSec 2004 at IEEE PerCom (2004)

2. Lee, S.M., Hwang, Y.J., Lee, D.H., Lim, J.I.: Efficient Authentication for Low-Cost RFID Systems. In: Gervasi, O., Gavrilova, M., Kumar, V., Laganà, A., Lee, H.P., Mun, Y., Taniar, D., Tan, C.J.K. (eds.) ICCSA 2005. LNCS, vol. 3483, Springer, Heidelberg (2005)

3. Weis, S.A., Sarma, S.E., Rivest, R.L., Engels, D.W.: Security and Privacy Aspects of LowCost Radio Frequency Identification Systems. In: International Conference on Security in Pervasive Computing (March 2003)

4. Li, Y.Z., Cho, Y.B., Um, N.K., Lee, S.H.: Security and Privacy on Authentication Protocol for Low-cost RFID. In: IEEE International Conference on Computational Intelligence and Security, vol. 2, pp. 1101-1104 (November 2006)

5. Lee, Y.K., Verbauwhede, I.: Secure and Low-cost RFID Authentication Protocols. Adaptive Wireless Networks-AWiN (November 2005)

6. Chien, H.Y., Chen, C.H.: Mutual Authentication Protocol for RFID Conforming to EPC Class 1 Generation 2 Standards. Computers Standards and Interfaces 29(2), 254-259 (2007)

7. Lin, C.-L., Chang, G.-G.: Cryptanalysis of EPC Class 1 Generation 2 RFID authentication. In: Information Security Conference 2007, ChiaYi, Taiwan (2007)

8. Duc, D.N., Park, J., Lee, H., Kim, K.: Enhancing Security of EPCglobal Gen-2 RFID Tag against Traceability and Cloning. In: The 2006 Symposium on Cryptography and Information Security (2006)

9. Karthikeyan, S., Nesterenko, M.: RFID security without extensive cryptography. In: Workshop on Security of ad hoc and sensor networks, pp. 63-67. ACM Press, Alexandria, Virginia, USA (2005) 